\section{PERINATAL HIV-1 INFECTION IN AN EXTREMELY PREMATURE INFANT}

Roukaya Al Hammoud, MD; Gloria Heresi, MD; James R Murphy, PhD; Norma Pérez, DO

McGovern Medical School, The University of Texas Health Science Center at Houston, Texas, USA

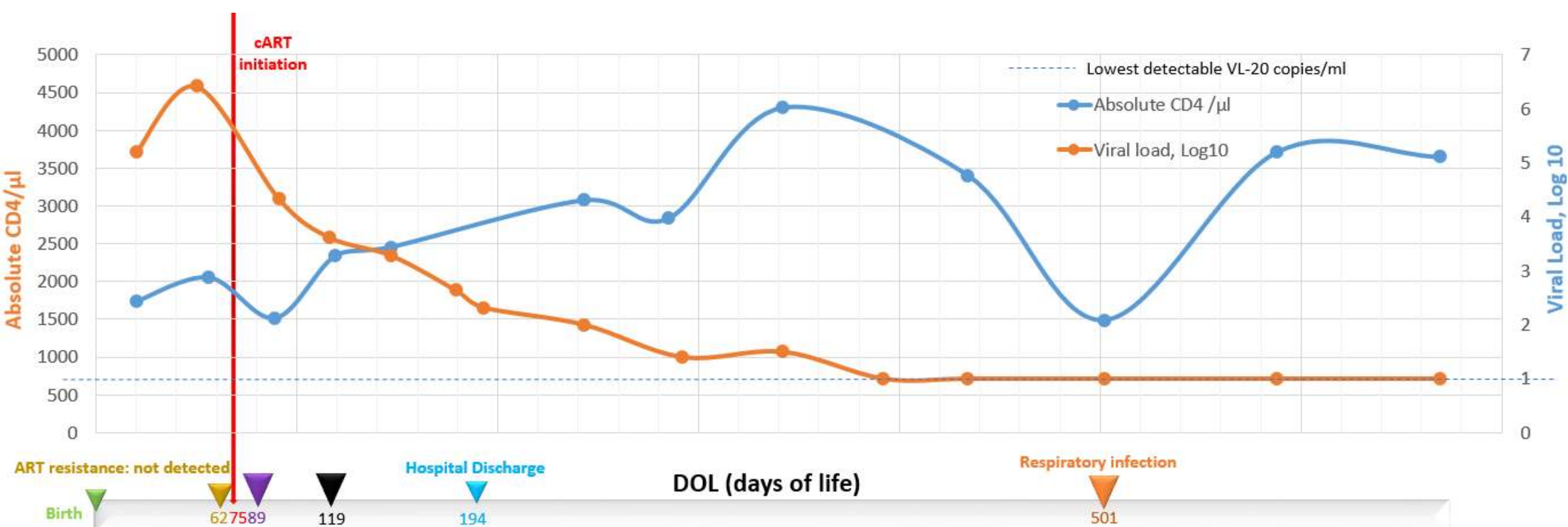

Background:

The rate of mother to child transmission (MTCT) of HIV in the United States has declined to $<1 \%$ thanks to multiple preventive strategies. Prematurity and HIV have a complex interaction. Maternal HIV is associated with prematurity. Prematurity is associated with a higher risk of MTCT in vaginal deliveries. Mortality rates are significantly high among HIV-infected infants born prematurely. Although there are substantial data supporting the benefits of combined antiretroviral medications (CART) initiation soon after birth, there are no clear recommendations on how to apply this to premature $\mathrm{HIV}+$ infants. Here, we describe an infant born at 24 weeks of gestation who was treated with early cART.

Case Description:

A female infant was born at 24 weeks of gestation to an $\mathrm{HIV}_{+}$mother with poor adherence to prescribed cART. Mother presented with rupture of the membranes and vaginal bleeding and infant was delivered via C-section. On the day of delivery, maternal viral load was 1240 copies $/ \mathrm{ml}$ and CD4 count was $792 / \mu l$. The patient's first 2 HIV tests were on day of life (DOL) 13 (qualitative HIV DNA PCR) and 21 (HIV RNA) and were positive. Patient had a viremia peak of 2,620,000 copies $/ \mathrm{ml}$. Patient received dual antiretroviral prophylaxis and was started on NVP-based CART on DOL 75. At corrected gestational age 42 weeks, infant was switched to LPN/r-based CART. ZDV was later switched to $A B C$ due to anemia. Because of the interaction between LPN $/ r$ and sildenafil (given for pulmonary hypertension), cART was switched to a RAL based one. Hospital course was complicated by bronchopulmonary dysplasia, pulmonary hypertension and pancreatic enzymes deficiency of unclear etiology (cystic fibrosis

Regimen 1

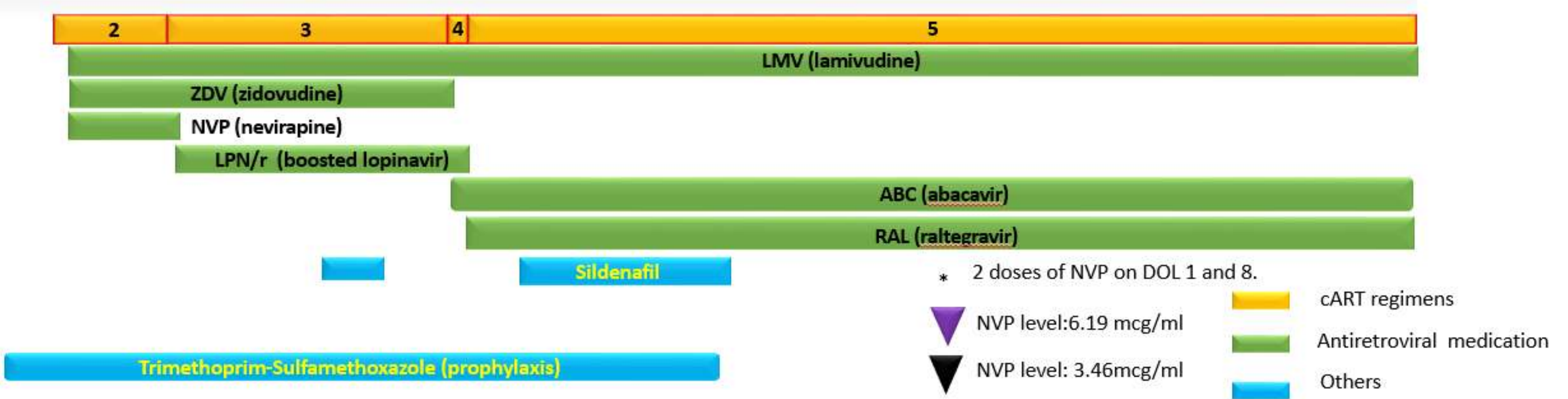

Figure: Timeline of laboratory, clinical and CART regimens. testing negative). Apart from being on oxygen therapy for chronic lung disease, infant continued to thrive well.

Discussion:

Mortality rates in premature HIV+ infants are significantly higher when compared to term infants (3-fold). Premature infants have immature immune response and inadequate skin/mucosal barrier allowing for more disease progression. Early cART can reduce infant mortality, disease progression (CHER trial, PMID 19020325) and magnitude of latent viral reservoir. It's also associated with better neurodevelopmental outcomes that can be adversely influenced by prematurity itself. For this, aggressive diagnostic and therapeutic measures in $\mathrm{HIV}+$ preemies seem important. This is, however, challenged by inadequacy of both strong evidence supporting early cART and available dosing recommendations of retroviral medications in this age group. Our other challenges include drug-drug interactions, lack of adequate drug level monitoring and morbidities related to clearance (>10 months) that can be related to malabsorption, other pharmacokinetics factors in young infants, and immature neonatal prematurity. Notably, the patient had slow viral immune system that is incapable of controlling HIV. Fortunately, the patient did not have any documented opportunistic or nosocomial bacterial/viral infections. CD4 count never dropped below $750 / \mu$ or $26 \%$ (Stage 3, CDC) Conclusions:

Although with many challenges, early cART initiation is possible in premature $\mathrm{HIV}_{+}$infants but more data are needed regarding safe dosing, benefits/risks and long term outcomes. This is crucial to avoid missing a potential window (soon after birth or confirmed diagnosis) for viral reservoir attenuation and better clinical outcomes. 